\title{
Homoepitaxial Nanostructures of Zinc Oxide
}

\author{
Tatiana V. Plakhova, ${ }^{1,2}$ Andrey N. Baranov, ${ }^{2}$ \\ Alexander V. Knotko, ${ }^{2}$ and Gennady N. Panin ${ }^{3,4}$ \\ ${ }^{1}$ Department of Material Sciences, Moscow State University, Leninskie Gory 3, Moscow 1199992, Russia \\ ${ }^{2}$ Department of Chemistry, Moscow State University, Leninskie Gory 3, Moscow 1199992, Russia \\ ${ }^{3}$ Physics Department, QSRC, Dongguk University, 3-26 Pildong, Junggu, Seoul 100-715, Republic of Korea \\ ${ }^{4}$ Institute for Microelectronics Technology of Russian Academy of Science, Chernogolovka 142432, Russia
}

Correspondence should be addressed to Andrey N. Baranov; anb@inorg.chem.msu.ru

Received 31 August 2015; Accepted 20 October 2015

Academic Editor: Giovanni Bongiovanni

Copyright (C) 2015 Tatiana V. Plakhova et al. This is an open access article distributed under the Creative Commons Attribution License, which permits unrestricted use, distribution, and reproduction in any medium, provided the original work is properly cited.

\begin{abstract}
The homoepitaxial $\mathrm{ZnO}$ nanostructures (HENS) were obtained on different substrates using various techniques. The first type of homoepitaxial $\mathrm{ZnO}$ nanorod arrays was grown on Si or ITO substrates by using two alternative sequences: (a) seeding $\rightarrow$ growth from solution $\rightarrow$ growth from vapor and contrariwise (b) seeding $\rightarrow$ growth from vapor $\rightarrow$ growth from solution. As follows from transport and cathode luminescence measurements homoepitaxial growth allows enhancing electrical or luminescence properties. The second type of HENS was prepared by growth of vertically or horizontally oriented $\mathrm{ZnO}$ nanorod arrays depending on monocrystalline $\mathrm{ZnO}$ wafers with [0001] and [10-10] orientation. In all cases the growth occurs along the $c$-axis of fast growth.
\end{abstract}

\section{Introduction}

Zinc oxide $(\mathrm{ZnO})$ is a unique functional semiconductor material with a wide band gap (3.37 eV), high binding energy of the exciton $(60 \mathrm{meV})$ at room temperature, and an effective ultraviolet luminescence $[1,2]$. Quasi-one-dimensional (1D) structures on the basis of zinc oxide are a promising material for nanoelectronics $[3,4]$. The array of nanorods has a high specific surface and crystalline quality with a high electron mobility along the nanorods. In contrast to the epitaxial films, the structure of nanorod arrays is free from strains thus being potentially interesting for optoelectronic applications in the blue/violet spectral range. A variety of synthetic techniques was developed, for example, (a) the growth from zinc containing vapor [5-7] and (b) the growth from zinc containing solutions [3, 8-11]. The optical and electronic transport properties of $\mathrm{ZnO}$ nanorods are significantly dependent on the synthesis condition, growth substrates, and other factors. For example, $\mathrm{ZnO}$ nanorods grown from vapor at relatively high temperature usually have excellent luminescent properties whereas $\mathrm{ZnO}$ nanorods grown from solution have better conductivity. Stable and reproducible p-type $\mathrm{ZnO}$ for fabricating $\mathrm{ZnO}$ homojunction optoelectronic devices still remains challenging. The majority of intrinsic defects are donors. When $\mathrm{ZnO}$ is doped with an acceptor, the compensation of donors takes place. The possibility of p-type doping strongly depends on the growth method, so for designing multilayer structures with $\mathrm{n}$ - and p-doped layers it is necessary to have a choice of the growth methods and to apply each of them aiming to reach the desired properties $[3,4]$.

There are only a few examples of epitaxial growth of $\mathrm{ZnO}$ nanostructures presented in the literature excluding numerous examples of film growth on various epitaxial substrates. For example, vertically well-aligned $\mathrm{ZnO}$ nanorods were grown on c-plane sapphire substrates coated with $\mathrm{ZnO}$ films by a vapor transport method and it was reported that the optical and electronic transport properties of $\mathrm{ZnO}$ nanowires are considerably influenced by roughness which is associated with the surface defects [12]. Highly uniform and $c$-axis-aligned $\mathrm{ZnO}$ nanorod arrays were fabricated by a low temperature homoepitaxial aqueous chemical method on the $\mathrm{Zn}$ - and $\mathrm{O}$-terminated single crystal $\mathrm{ZnO}$ wafers. The effect of the surface polarity was also examined; however, no 
significant difference was found between the arrays grown on $\mathrm{Zn}$-terminated and on O-terminated face of the $\mathrm{ZnO}$ single crystal [13]. The possibility of fabricating arrays of densely located, ordered $\mathrm{ZnO}$ nanopillars on commercially available low cost $\mathrm{Si}$ substrates by atmospheric pressure metal organic chemical vapor deposition (APMOCVD) via selective homoepitaxial growth was shown. The grown $\mathrm{ZnO}$ nanopillars demonstrated a noticeable light emitting ability at room temperature, possessing an intense peak of UV emission, and absence of defect luminescence in the visible range of spectra [14]. Array-oriented $\mathrm{ZnO}$ nanocrystals were synthesized on the surface of gallium nitride [0001] by the hydrothermal growth [15]. Excellent matching between the lattice parameters of $\mathrm{GaN}$ and $\mathrm{ZnO}$ promotes epitaxial nucleation at the substrate of gallium nitride. The formation of a nucleus at the surface of the substrate has a lower energy barrier than the formation of the nucleus in the volume of the reaction mixture, so the growth of oriented nanorods at the GaN substrates (and other epitaxial substrates) is possible without preliminary seeding. For epitaxial growth of nanorods by chemical vapor deposition using metalorganic precursors zinc oxide substrates also could be used. In this instance, the nanorods can grow with a minimum concentration of misfit dislocations at the substrate, nanorod interface. Epitaxial growth in axial and biaxial directions is possible only if the substrate is smooth (the radius of curvature in some parts of the surface shall not exceed $1.0 \mathrm{~nm}$ ) [16]. The surface roughness of the substrate prevents the epitaxial growth of $\mathrm{ZnO}$ nanorods. According to the authors in [16] stress caused by the mismatch of the planes nanorod and the substrate is the main reason for nonepitaxial growth of nanocrystals on rough substrates. Much less frequently in the literature one can find examples of the epitaxial growth on the surface of the nanorods themselves. We found only two examples of this growth: the growth of nanorods on the surface of nanocones [17] and the growth of $\mathrm{ZnO}$ films on the tips of the nanorods, forming a uniform array [18].

The development of optoelectronic devices requires (a) high quality p-type and n-type layers; (b) high quality interface between layers; and (c) excellent luminescent and transport properties of layers. These problems potentially could be solved by application of various growth methods and substrates. Thus, the aims of the work were the synthesis and the study of the morphology and properties of homoepitaxial $\mathrm{ZnO}$ nanostructures (HENS). HENS were prepared by consecutive usage of two different growth methods: growth from solution and vapor phase. Another kind of HENS was obtained by the growth of $\mathrm{ZnO}$ nanorods at zinc oxide monocrystalline wafers with [0001] and [10-10] orientation.

\section{Experimental Section}

Monocrystalline silicon wafers, ITO, and monocrystalline $\mathrm{ZnO}$ wafers with [0001] and [10-10] orientation (produced by "Mineral Ltd.", Alexandrov, Russia) were used in the present work as a substrate for homoepitaxial $\mathrm{ZnO}$ nanorods growth. Before the synthesis $\mathrm{ZnO}$ substrates were immersed into acetone, sonicated by ultrasound for 10 minutes, and rinsed by distilled water. $\mathrm{Si}$ and ITO substrates before $\mathrm{ZnO}$ growth were cleaned by $10 \%$ hydrochloric acid and rinsed by distilled water.

To produce a uniform seed layer, we used a $0.005 \mathrm{M}$ $\mathrm{Zn}\left(\mathrm{CH}_{3} \mathrm{COO}\right)_{2} 2 \mathrm{H}_{2} \mathrm{O}$ solution in ethanol (abs., 99,95\%). A drop of the solution was applied to a substrate ( $\mathrm{Si}$ or ITO) rotating at $500 \mathrm{rpm}$. After ethanol vaporization, the substrate surface had a uniform zinc acetate layer, which was decomposed to $\mathrm{ZnO}$ seed layer at temperatures of $350^{\circ} \mathrm{C}$ for $2 \mathrm{~h}[8]$.

Chemical bath deposition was carried out in a reaction beaker using $3 \mathrm{~mL}$ of a $20 \%$ ethylenediamine $\mathrm{NH}_{2} \mathrm{CH}_{2} \mathrm{CH}_{2} \mathrm{NH}_{2}(\geq 99,9 \%)$ and $0.2 \mathrm{M}$ zinc acetate aqueous solutions. Substrates with $\mathrm{ZnO}$ seeds were immersed in the mixture of solutions. The reaction vessel was tightly closed and placed for $2 \mathrm{~h}$ in an oven maintained at $105-110^{\circ} \mathrm{C}$ for 1.5 hours. After the synthesis reached completion, the samples were carefully washed with distilled water and dried in air.

For the growth zinc oxide from gas phase a quartz reactor with a boat with zinc powder $(99,99 \%)$ precursor and pretreated substrates were placed in a two-zone tube furnace [8]. The reactor was vacuum pumped for $1 \mathrm{~h}$. Next, argon flow was admitted to the reactor, and the temperature in the first and second zones was increased up to their working temperatures, 620 and $570^{\circ} \mathrm{C}$, respectively. After that, oxygen flow was released into the system. The fraction of oxygen in the gas mixture was $25 \mathrm{vol} \%$. The duration of synthesis was 1 hour.

Homoepitaxial nanostructures were prepared by the following methods. First, zinc oxide nanorods were grown from the gas phase on the top of nanorods preliminarily synthesized by chemical bath deposition (HENS_1). Secondly, the growth of nanorods was carried out from the solution on the top of nanorods preliminarily grown from the gas phase (HENS_2). Another type of nanostructure was grown on the monocrystalline $\mathrm{ZnO}$ wafers by the chemical bath or gas phase deposition. The orientations of crystallographic axes were [0001] and [10-10]. Table 1 lists the corresponding names of the sample and the growth conditions.

The morphology of the samples was examined by scanning electron microscopy (SEM) on a LEO Supra 50 VP. Transmission electron microscopy (TEM) studies were carried out using a transmission electron microscope JEM 2000FXII (JEOL, Japan) at accelerating voltage of $200 \mathrm{kV}$. The electron diffraction pattern from the selected area was obtained using 0.3-micron selector diaphragm. The structural measurements for the samples were performed by X-ray diffraction (XRD) using Rigaku D/MAX 2500 diffractometer $\left(\mathrm{Cu}_{\mathrm{K} \alpha}\right.$ radiation $(\lambda=1.54184 \AA)$, rotating anode $\mathrm{X}$-ray tube). The decomposition temperature of zinc acetate was determined by differential thermal analysis (DTA) and thermogravimetric analysis (TGA) using a PerkinElmer Model Diamond TG/DTA thermoanalytical system. Cathodoluminescence (CL) spectra were collected at room temperature using XL 30S FEG high-resolution scanning electron microscope with a mono-CL system in the 300-900 nm wavelength range. The AFM study of $\mathrm{ZnO}$ single crystal's surface was performed under ambient conditions using N-TEGRA scanning probe microscope (NT MDT production) equipped with commercial Si cantilevers NSC14. 
TABLE 1: Homoepitaxial nanostructures synthesized in this work.

\begin{tabular}{lcc}
\hline Sample & Substrate & Synthesis \\
\hline HENS_1 & Si or ITO & Seeding $\rightarrow$ growth from solution $\rightarrow$ growth from vapor \\
HENS_2 & Si or ITO & Seeding $\rightarrow$ growth from vapor $\rightarrow$ growth from solution \\
GF_0001 & Monocrystalline ZnO wafer [000-1] orientation & Growth from vapor \\
S_0001 & Monocrystalline ZnO wafer [0001] orientation & Growth from solution \\
S_10-10 & Monocrystalline ZnO wafer ZnO [10-10] orientation & Growth from solution \\
\hline
\end{tabular}

\section{Results and Discussion}

3.1. Nanostructures HENS_1 and HENS_2. Figure 1 shows micrographs of the HENS_1 (Figure 1(b)) and HENS_2 (Figure 1(d)) samples. Figures 1(a) and 1(c) present $\mathrm{ZnO}$ nanorods preliminarily grown from solution (a) and (c) gas phase. In both cases, height of nanorods was increased on a few micrometers in accordance with growth rate at applied experimental conditions. Also one can see noticeable increase in thickness at the bottom of the rods but nanorods growth occurs mainly along the 0001 axis of rapid growth. HENS nanostructures were studied by X-ray analysis. Figure 1(f) shows diffraction pattern of HENS_2 in the given range of angles $\left(\theta=10-60^{\circ}\right)$ where there is only one reflection corresponding to the interplanar distance of 002 . This reflex relates to a zinc oxide phase. No other reflections indicate the absence of impurities in the sample and excellent orientation in the 0001 direction. The XRD rocking curve of the (0002) peak obtained from the sample is shown in Figure 1(e). The full-width half-maximum (FWHM) reflects the small angular dispersion of the crystallites around the $c$-axis.

Using TEM and selected area electron diffraction (SAED) techniques (Figures 2(a)-2(h)) we found the identity of the diffraction from different region of a single nanorod with the same position of microscope goniometer and specimen holder. Therefore, HENS_1 and HENS_2 samples are single crystal without interface boundary; the growth of structures is epitaxial not only in $c$-axis but also in $a$-and $b$-axis directions.

On the cathodoluminescence spectra (Figure 3), we can observe several peaks. The narrow intense peak around $383 \mathrm{~nm}$ corresponds to the recombination of free excitons. Emission in the range of $500-550 \mathrm{~nm}$ region is most often associated with the deep impurity levels such as oxygen vacancies, zinc interstitials, and recombination of donoracceptor pairs. Usually the high intensity ratio $(I x / I g)$ of the UV and visible luminescence indicates the absence of impurities and related defects. During the synthesis from solution, in addition to deep intrinsic defects, impurities at the level of $0.01-0.1 \%$ (classification used reagent grade chemicals) appear. Therefore, the samples prepared from solution exhibit a broad intense peak in green range of spectra (Figure 3(a)). Synthesis from the vapor phase is carried out at rather high temperature with the use of high-purity precursors ( $\mathrm{Zn}$ metal) and gases. Thus intensive emission in the UV range is observed in the spectrum (Figure 3(b)).

Figure 3(b) shows the spectrum of the cathodoluminescence of HENS_2 sample. Compared with the previously synthesized array grown from the gaseous phase, an intensity of the exciton luminescence ( $I x)$ decreases and a broad peak in the green range $(I g)$ appears. The quality index of nanorods significantly decreases: from $I x / I g=22$ to $I x / I g=$ 0.05. The green luminescence peak for the sample HENS_1 (Figure 3(a)) is considerably suppressed in comparison with the exciton one. This fact could be explained by the effect of annealing pregrown sample during the vapor phase synthesis of HENS_1 structure.

The current-voltage dependence for the HENS_1 and HENS_2 structures along the growth direction of the nanorods shows linear current-voltage characteristics indicating an ohmic behavior of resistance. Values specific resistivity $\rho$ of the $\mathrm{ZnO}$ nanostructures (HENS_1) $=2 \mathrm{kOhm} *$ $\mathrm{cm}, \rho$ (HENS_2) $=600 \mathrm{kOhm} * \mathrm{~cm}$ has been estimated by the formula: $\rho=R * S / L$, where $R$ is resistance of the nanostructure measured along the direction of the $c$-axis, $S$ is the effective area of contact, and $L$ is length of the nanorods. Difference in the resistivity of homoepitaxial nanostructures HENS_1 and HENS_2 can be explained by their preparation prehistory. According to the literature data $[3,8,9]$ and our own data, the specific resistivity of nanorods grown from a solution is relatively low $(\rho=\sim 500 \mathrm{Ohm} * \mathrm{~cm})$ because of the presence of a large number of shallow impurity levels. Transport properties of HENS_2 nanostructure are mostly determined by the properties of nanorods grown from solution whereas transport properties of HENS_1 nanostructure are determined by the properties of the nanorods grown from the gas phase.

\subsection{The Growth of Nanorods at the Single Crystal Substrates}

3.2.1. Homoepitaxial Vertical Growth of Nanorod Arrays on Zinc Oxide Substrate. Interesting morphology of $\mathrm{ZnO}$ nanorods was observed after the deposition from the gas phase on the $\mathrm{ZnO}$ single crystal substrate with crystal orientation in the [0001] direction (zinc polar side): a pyramidal base with nanorods growing out of its top (Figures 4(a) and 4(b)). Height of the basement of the pyramids is around $2 \mu \mathrm{m}$, and nanorod height is $2 \mu \mathrm{m}$. From the top view, we can estimate the diameter of nanorods as $100 \mathrm{~nm}$. Basement of pyramid ( $2 \mu \mathrm{m}$ at the bottom and $450 \mathrm{~nm}$ at the top) is presented in the form of hexagon and the sides of one hexagon are parallel to the sides of other hexagons. This fact proves the epitaxial growth of the pyramids along the crystallographic directions $a b$. Epitaxial growth in the axial direction is confirmed by the orientation of the pyramids and a rod at an angle of $90^{\circ}$ to the substrate surface. 


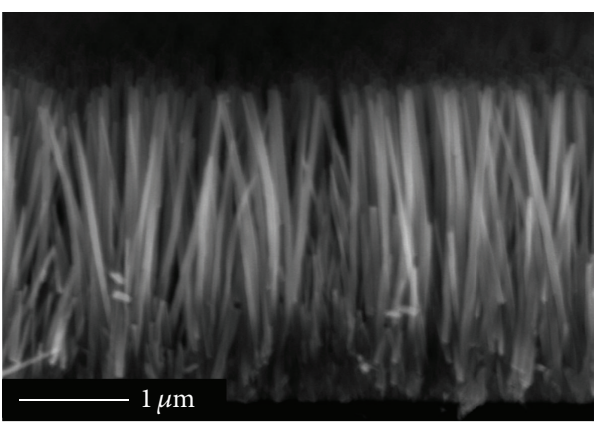

(a)

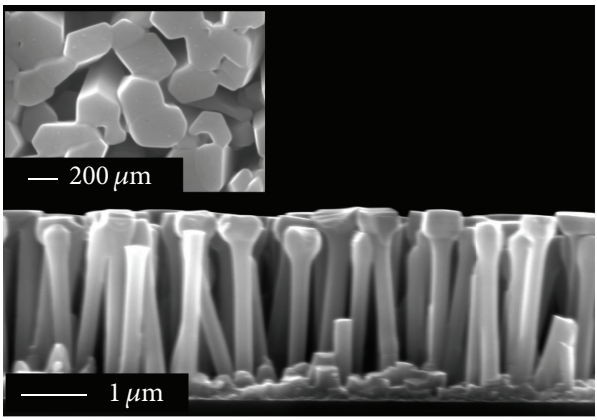

(c)

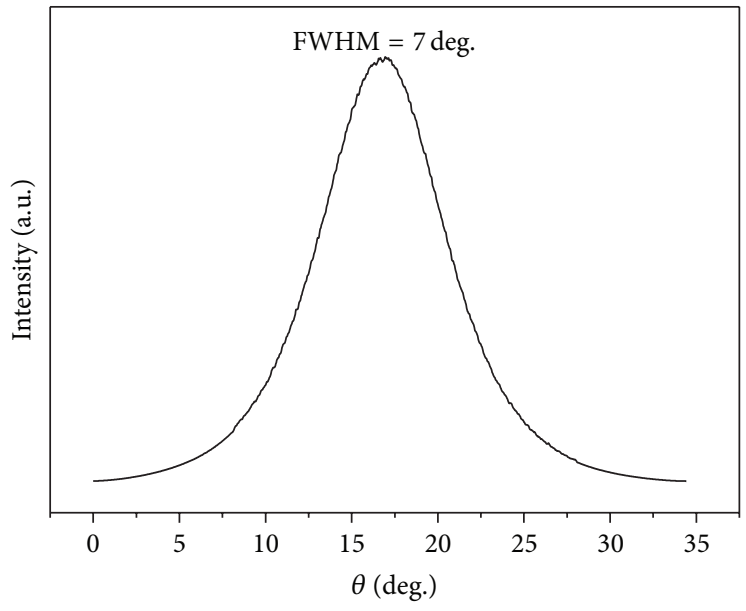

(e)

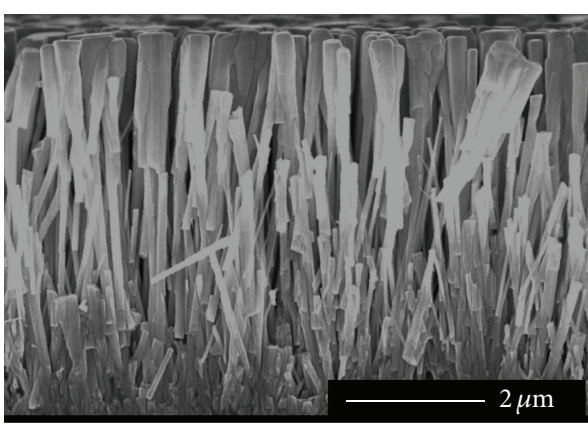

(b)

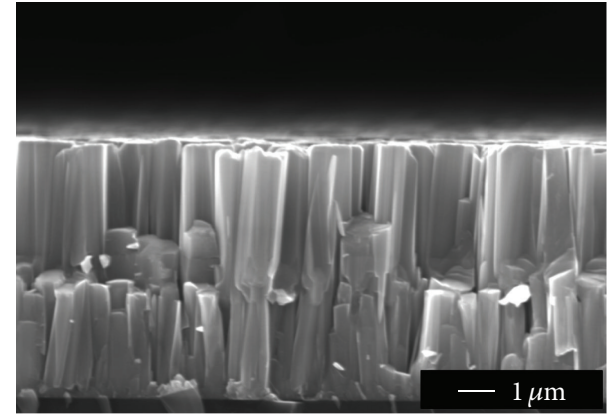

(d)

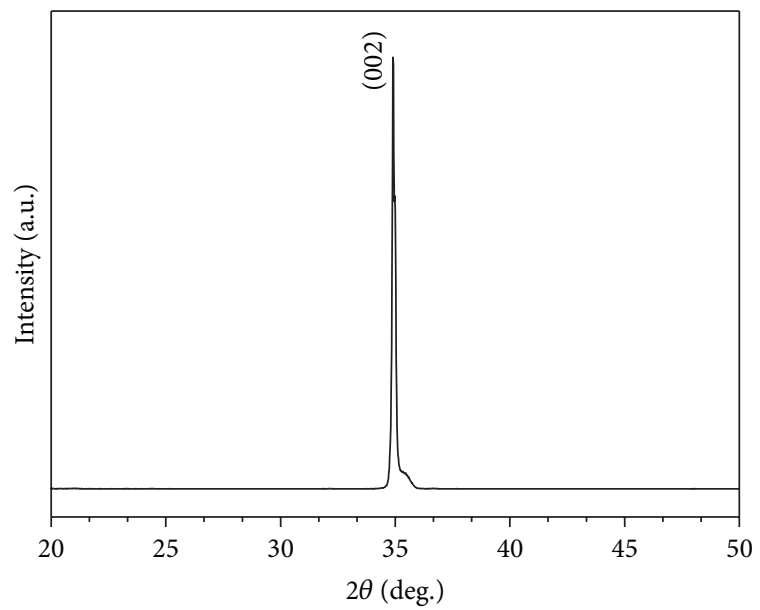

(f)

FIGURE 1: SEM images of (a) sample grown from solution, (b) nanostructure HENS_1, (c) side view and (insert) top view of gas phase sample, and (d) nanostructure HENS_2. (e) XRD rocking curve of the (0002) peak of HENS_1 sample; (f) diffraction pattern of HENS_2 sample.

A similar morphology of the samples, grown on oxygen polar side of $\mathrm{ZnO}$ wafer, was described in the work [19]. According to the backscattered electron diffraction direction of the nanorods growth coincides with the direction 0002, and $\mathrm{ZnO}$ nanorods have $\mathrm{Zn}$-polar surface. At the same time the growth of the pyramidal base occurs along the direction of 000-2; and pyramids are O-polar. Based on the results of electron diffraction, the authors of the work [19] argue that the opposite direction of growth leads to different morphological pyramids or nanorods. During the growth of nanorods on $\mathrm{Zn}$-polar crystals, at the initial stages, the O-polar buffer layer is formed, and then the growth of the above-described structures continues. In the case of our samples the drop-like features at the substrate (Figure 4(a)) can be identified as buffer layer. Figure 4(c) shows the cathodoluminescence spectrum of the sample GF_0001 (nanorods grown from the gas phase at the surface of a single crystal with the orientation of [0001]). Other spectra are the local cathodoluminescence spectra and were collected from the nanorods and the substrate. In all cases, there is only one intensive UV peak (Figure 4(c)). The intensity of the exciton peak of nanorods coincides with that of the single crystal substrate one. This observation can be explained by the fact that the $\mathrm{ZnO}$ nanorods epitaxially grown at the surface of monocrystalline $\mathrm{ZnO}$ wafer inherit its defect structure. Figure 4(d) presents results of SEM for 


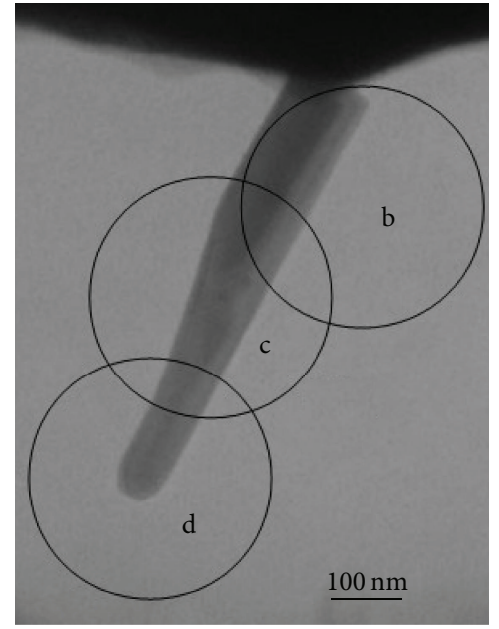

(a)

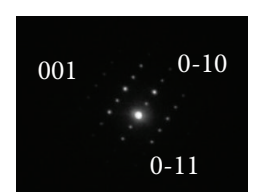

(b)

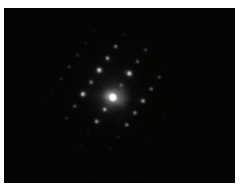

(c)

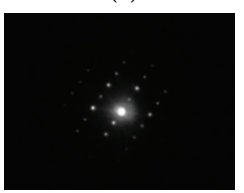

(d)

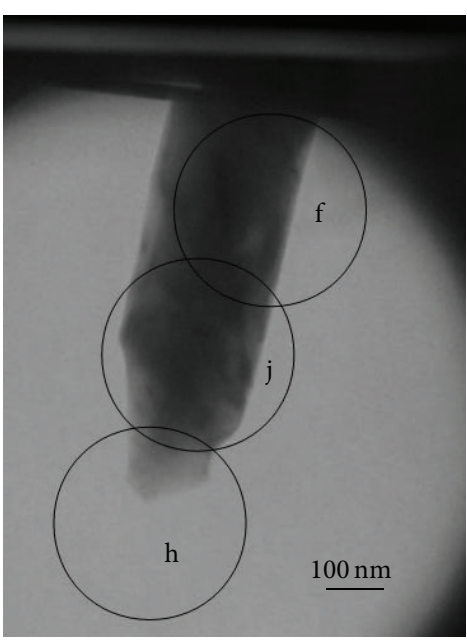

(e)

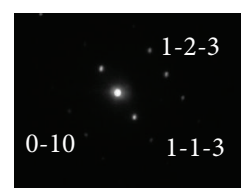

(f)

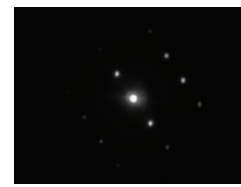

(j)

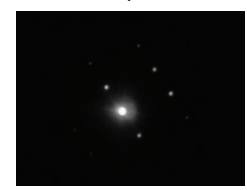

(h)

FIGURE 2: TEM images of (a) HENS_1 and (e) HENS_2 structures. [(b)-(d)], [(f)-(h)] SAED pattern from different parts of the homoepitaxial rods denoted by the circle regions.

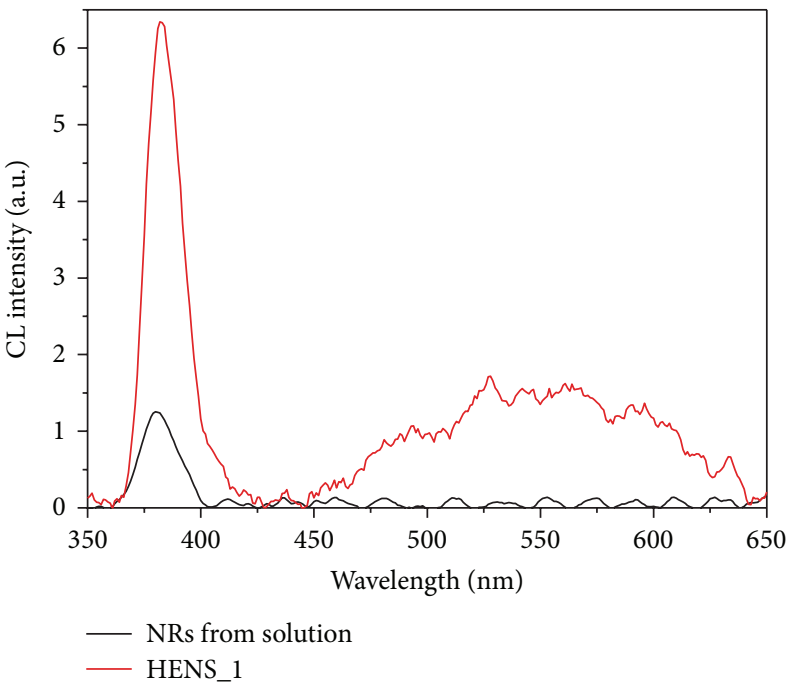

(a)

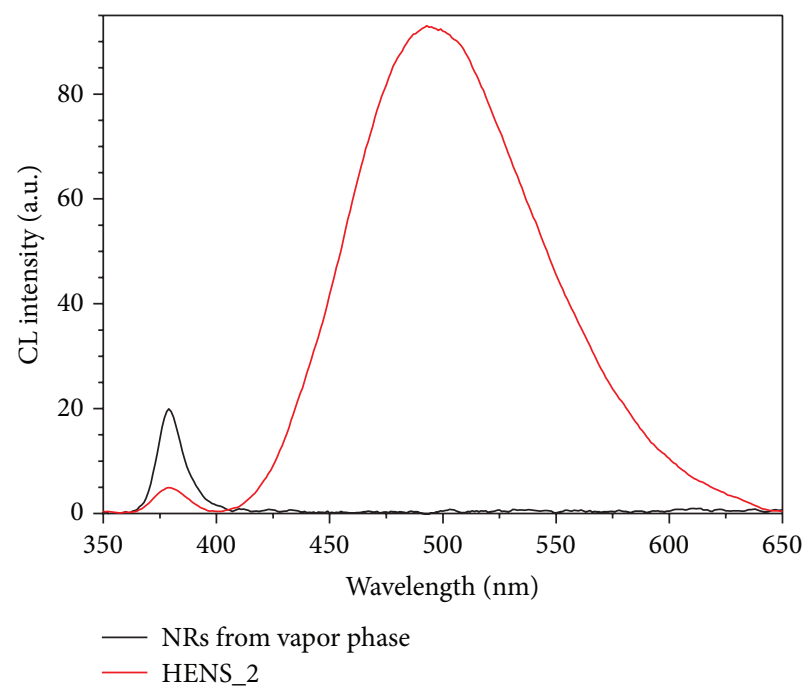

(b)

FIGURE 3: Cathodoluminescence spectra of (a) nanorods from solution and HENS_1 structure and (b) nanorods from vapor phase and HENS_2 structure.

the sample S_0001. Nanorods were grown at the $\mathrm{ZnO}$ wafer with orientation 0001 from the solution without seeding. The use of monocrystalline $\mathrm{ZnO}$ wafer results in a dense array of vertically oriented nanorods with the height of the array of $3 \mu \mathrm{m}$. Thus, SEM data confirm homoepitaxial growth of $\mathrm{ZnO}$ nanorods at monocrystalline $\mathrm{ZnO}$ wafer in the axial direction.

\subsubsection{Planar Growth of Nanorods on Zinc Oxide Substrate.} The vertical growth of $\mathrm{ZnO}$ nanorods has been rather well studied and it is possible to find enough literature on the subject. At the same time, horizontal growth or growth in a plane parallel to the substrate is practically not described in the literature. It is worth mentioning the work [20], where planar nanostructures were prepared by multistage synthesis with usage of lithography techniques. Alternatively, in this paper we present new method for growth of horizontally oriented $\mathrm{ZnO}$ nanorods structure by usage of monocrystalline $\mathrm{ZnO}$ wafer with orientation [10-10]. Direction of the rapid growth of nanorods and the orientation of the substrate in this experiment are not the same and this leads to the change of growth direction. Growth of nanorods occurred with a small angle of deviation from the horizontal direction to the surface. Nanorods length was approximately $6 \mu \mathrm{m}$ and nanocrystals have clearly visible hexagonal facets (Figures 5(a) and 5(b)). Our explanation for the observed experimental data is proposed in Figure 5(c). Screw dislocation at the surface of the crystal opens the plane of rapid growth 


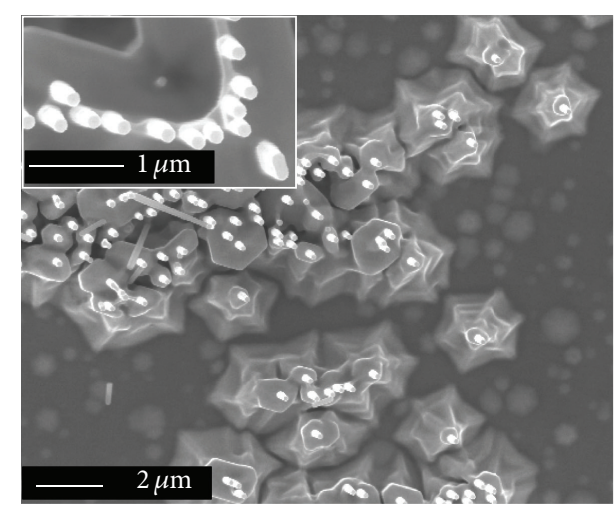

(a)

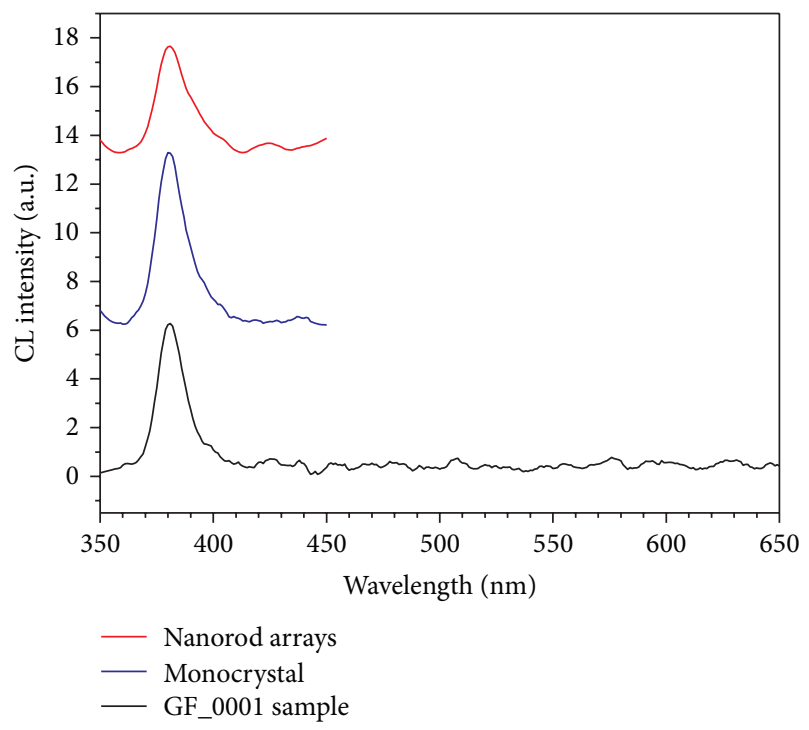

(c)

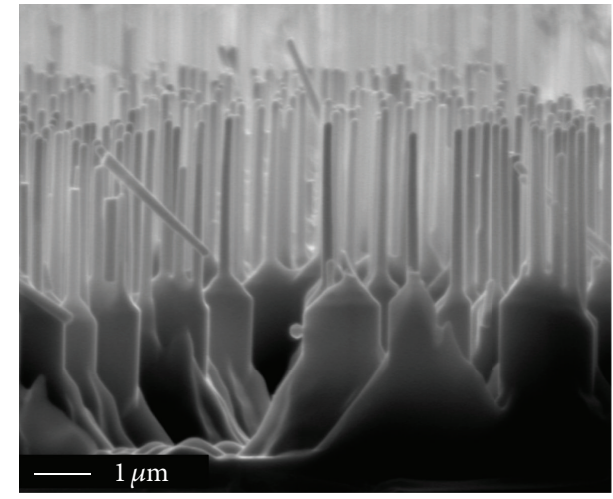

(b)

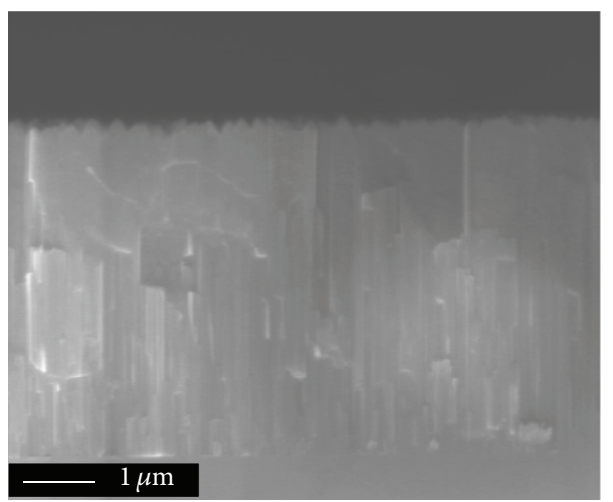

(d)

FIGURE 4: SEM micrographs of ZnO nanorods and pyramids: (a) top view and (b) side view. (c) Cathodoluminescence spectra of different parts of GF_0001 sample. (d) SEM data of S_0001 sample.

[0001] wurtzite, so growth occurs along the axis $c$ but is not perpendicular with very small angle zincite surface. According to the SEM (top view of the array) all the rods are aligned in the [10-10] plane. This fact suggests epitaxial growth of dislocation steps not only in $c$-axis direction but also in the directions of the axes $a b$ zincite. Concentration of dislocation can be estimated from the pitches after etching.

Besides the defects generated in the crystal during growth, there are different kinds of scratches (depth of about $40 \mathrm{~nm}$ and width of $300 \mathrm{~nm}$ ) on the wafer's surface appearing after mechanical polishing (Figure 5(d)). Scratches as well as screw dislocations open face [0001] and the corresponding direction, which is the direction of rapid growth. Therefore, for sample S_10-10 we can see not only the individual nanorods grown on screw dislocations, but also the aligned "fence," where the density of nanorods is rather high (Figure 5(b)). Comparing AFM data of empty [10-10] face with SEM data of the same surface after nanorods synthesis we can observe intensive population of nanorods regarding the original scratches on the substrate. The fact that the rods grow in the same direction regardless of the angle of the scratches also confirms homoepitaxial nature nanorod growth on single crystal zinc oxide substrates.

\section{Conclusions}

By combining the vapor phase and solution synthesis techniques vertical nanorod arrays were grown and the growth occurs in the $c$-axis direction. Luminescence of arrays of nanorods is better for the samples obtained by gas phase method whereas conductivity is higher in the case of solution method of growth. Solution technique leads to a better conductivity, whereas gas phase growth leads to more intense ultraviolet luminescence. The growth of nanorods from a solution on the [0001] monocrystalline $\mathrm{ZnO}$ wafer leads to the continuous epitaxial layer of zinc oxide and on the [000-1] facet we observed the formation of pyramidal bases with nanorods on top. These structures grow epitaxially on a substrate in the axial directions. In the case of monocrystalline 


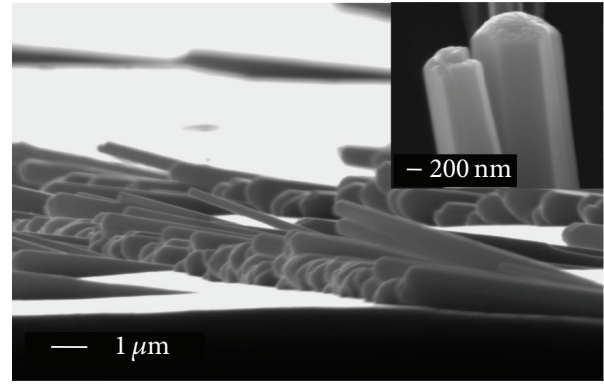

(a)

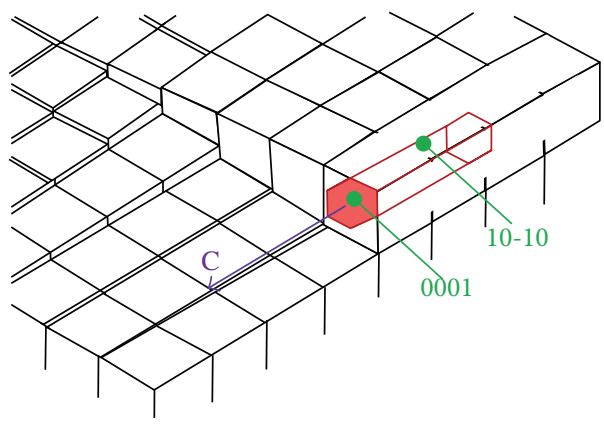

(c)

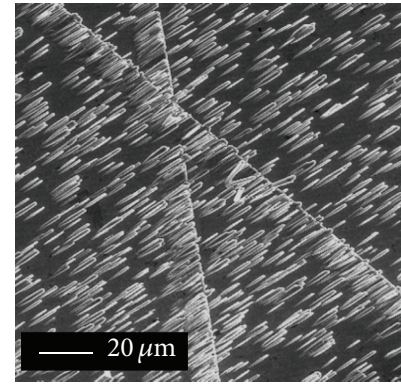

(b)

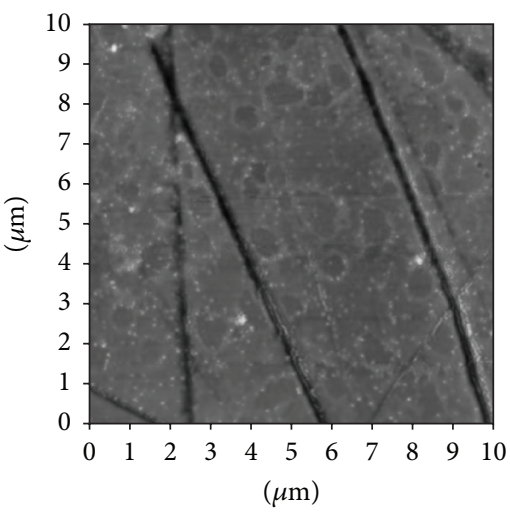

(d)

Figure 5: (a, b) SEM images of planar ZnO HENS. (c) Possible mechanism of planar growth on screw dislocations. The black arrowhead indicates the direction of rapid growth of the nanorod. (d) AFM data of zinc oxide [10-10] monocrystal surface.

$\mathrm{ZnO}$ wafer with the orientation [10-10] growth of the nanorods occurs in the planar direction.

\section{Abbreviations}

HENS: Homoepitaxial nanostructures.

\section{Conflict of Interests}

The authors declare no competing financial interests.

\section{Acknowledgments}

The authors would like to thank Vasily Lebedev for AFM measurements and also the company Mineral Ltd. for the kindly provided $\mathrm{ZnO}$ single crystal substrates (http://www syntecrys.com). This work was supported by the Russian Foundation for Basic Research (Project no. 14-03-00721).

\section{References}

[1] Ü. Özgür, Y. I. Alivov, C. Liu et al., "A comprehensive review of $\mathrm{ZnO}$ materials and devices," Journal of Applied Physics, vol. 98, no. 4, Article ID 041301, 2005.

[2] C. Klingshirn, J. Fallert, H. Zhou et al., "65 years of $\mathrm{ZnO}$ research-old and very recent results," Physica Status Solidi (B), vol. 247, no. 6, pp. 1424-1447, 2010.
[3] S. Xu and Z. L. Wang, "One-dimensional $\mathrm{ZnO}$ nanostructures: solution growth and functional properties," Nano Research, vol. 4, no. 11, pp. 1013-1098, 2011.

[4] D. Panda and T.-Y. Tseng, "One-dimensional ZnO nanostructures: fabrication, optoelectronic properties, and device applications," Journal of Materials Science, vol. 48, no. 20, pp. 6849-6877, 2013.

[5] P.-C. Chang, Z. Fan, D. Wang et al., "ZnO nanowires synthesized by vapor trapping CVD method," Chemistry of Materials, vol. 16, no. 24, pp. 5133-5137, 2004.

[6] W. D. Yu, X. M. Li, X. D. Gao, P. S. Qiu, W. X. Cheng, and A. L. Ding, "Effect of zinc sources on the morphology of $\mathrm{ZnO}$ nanostructures and their photoluminescence properties," Applied Physics A, vol. 79, no. 3, pp. 453-456, 2004.

[7] A. N. Red'kin, Z. I. Makovei, A. N. Gruzintsev, E. E. Yakimov, O. V. Kononenko, and A. A. Firsov, "Elemental vapor-phase synthesis of nanostructured zinc oxide," Inorganic Materials, vol. 45, no. 11, pp. 1246-1251, 2009.

[8] T. V. Plakhova, M. V. Shestakov, and A. N. Baranov, "Effect of textured seeds on the morphology and optical properties of solution- and vapor-grown $\mathrm{ZnO}$ nanorod arrays," Inorganic Materials, vol. 48, no. 5, pp. 469-475, 2012.

[9] O. V. Troshyn, A. A. Kovalenko, S. G. Dorofeev, and A. N. Baranov, "Sensitization of $\mathrm{ZnO}$ nanorods with CdSe quantum dots," Inorganic Materials, vol. 48, no. 7, pp. 709-715, 2012.

[10] K. Govender, D. S. Boyle, P. B. Kenway, and P. O'Brien, "Understanding the factors that govern the deposition and morphology of thin films of $\mathrm{ZnO}$ from aqueous solution," Journal of Materials Chemistry, vol. 14, no. 16, pp. 2575-2591, 2004 . 
[11] M. Guo, P. Diao, and S. Cai, "Hydrothermal growth of wellaligned $\mathrm{ZnO}$ nanorod arrays: dependence of morphology and alignment ordering upon preparing conditions," Journal of Solid State Chemistry, vol. 178, no. 6, pp. 1864-1873, 2005.

[12] W.-K. Hong, G. Jo, M. Choe, T. Lee, J. I. Sohn, and M. E. Welland, "Influence of surface structure on the phononassisted emission process in the $\mathrm{ZnO}$ nanowires grown on homoepitaxial films," Applied Physics Letters, vol. 94, no. 4, Article ID 043103, 2009.

[13] J. Volk, T. Nagata, R. Erdélyi et al., "Highly uniform epitaxial $\mathrm{ZnO}$ nanorod arrays for nanopiezotronics," Nanoscale Research Letters, vol. 4, no. 7, pp. 699-704, 2009.

[14] U. Pal and P. Santiago, "Controlling the morphology of $\mathrm{ZnO}$ nanostructures in a low-temperature hydrothermal process," The Journal of Physical Chemistry B, vol. 109, no. 32, pp. 1531715321, 2005.

[15] H. Q. Le, S. J. Chua, Y. W. Koh, K. P. Loh, and E. A. Fitzgerald, "Systematic studies of the epitaxial growth of single-crystal $\mathrm{ZnO}$ nanorods on GaN using hydrothermal synthesis," Journal of Crystal Growth, vol. 293, no. 1, pp. 36-42, 2006.

[16] C. Kwak, B.-H. Kim, C.-L. Park et al., "Microstructural properties at the interfaces of zno nanorods and ZNO homo-buffer layers," Journal of Nanoscience and Nanotechnology, vol. 10, no. 2, pp. 912-918, 2010.

[17] X. Han, G. Wang, L. Zhou, and J. G. Hou, "Crystal orientationordered $\mathrm{ZnO}$ nanorod bundles on hexagonal heads of $\mathrm{ZnO}$ microcones: epitaxial growth and self-attraction," Chemical Communications, no. 2, pp. 212-214, 2006.

[18] S.-H. Park, S.-Y. Seo, S.-H. Kim, and S.-W. Han, "Homoepitaxial $\mathrm{ZnO}$ film growth on vertically aligned $\mathrm{ZnO}$ nanorods," Journal of Crystal Growth, vol. 303, no. 2, pp. 580-584, 2007.

[19] G. Perillat-Merceroz, R. Thierry, P.-H. Jouneau, P. Ferret, and G. Feuillet, "Compared growth mechanisms of $\mathrm{Zn}$-polar $\mathrm{ZnO}$ nanowires on O-polar ZnO and on sapphire," Nanotechnology, vol. 23, no. 12, Article ID 125702, 2012.

[20] Z. L. Wang, R. Yang, J. Zhou et al., "Lateral nanowire/nanobelt based nanogenerators, piezotronics and piezo-phototronics," Materials Science and Engineering R: Reports, vol. 70, no. 3-6, pp. 320-329, 2010. 

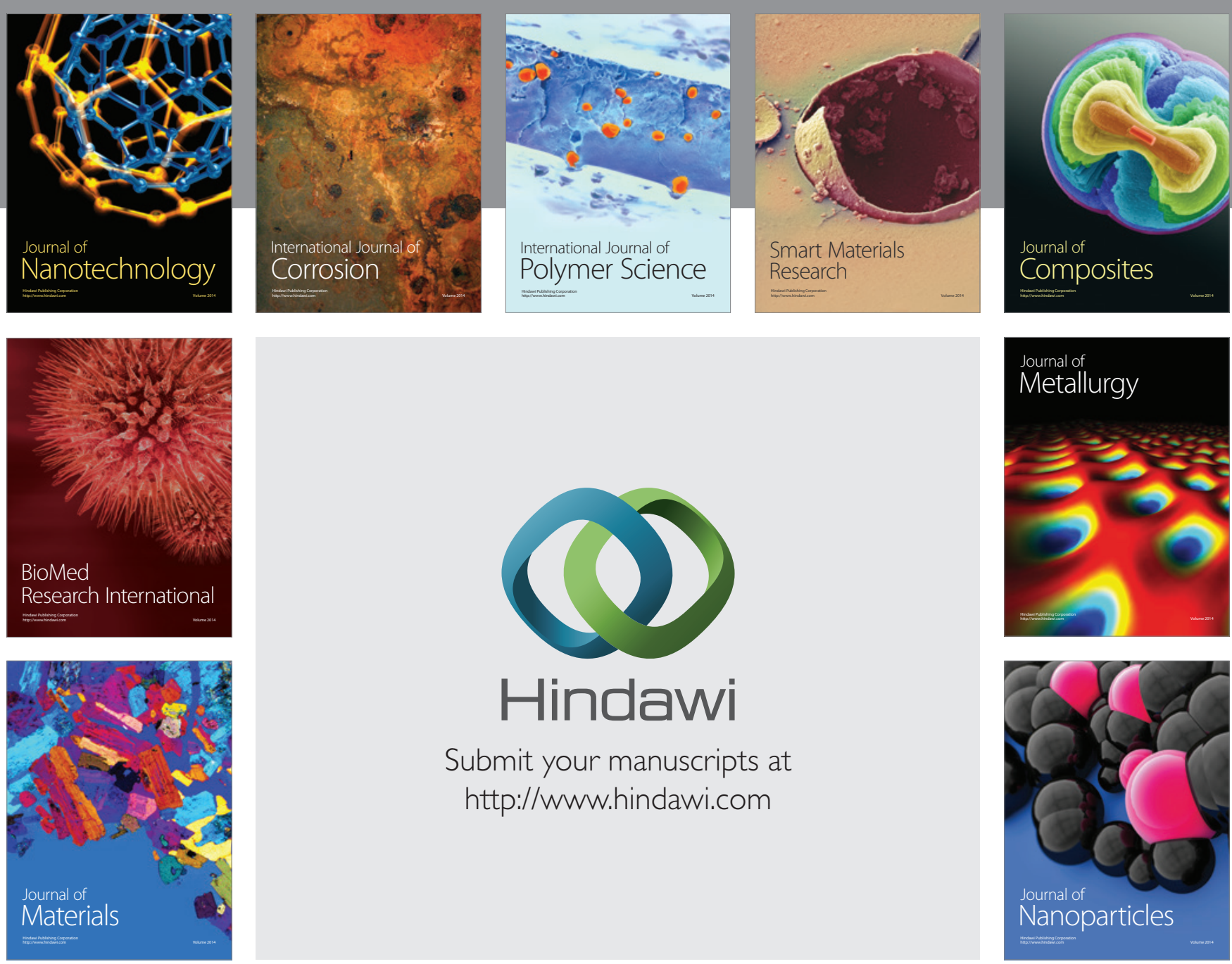

Submit your manuscripts at http://www.hindawi.com
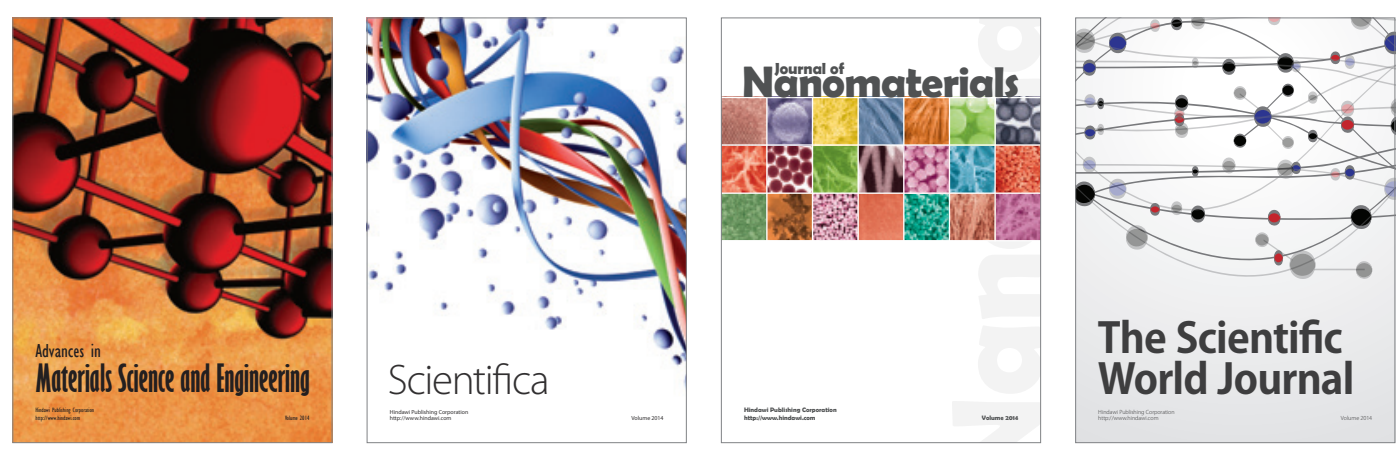

\section{The Scientific World Journal}
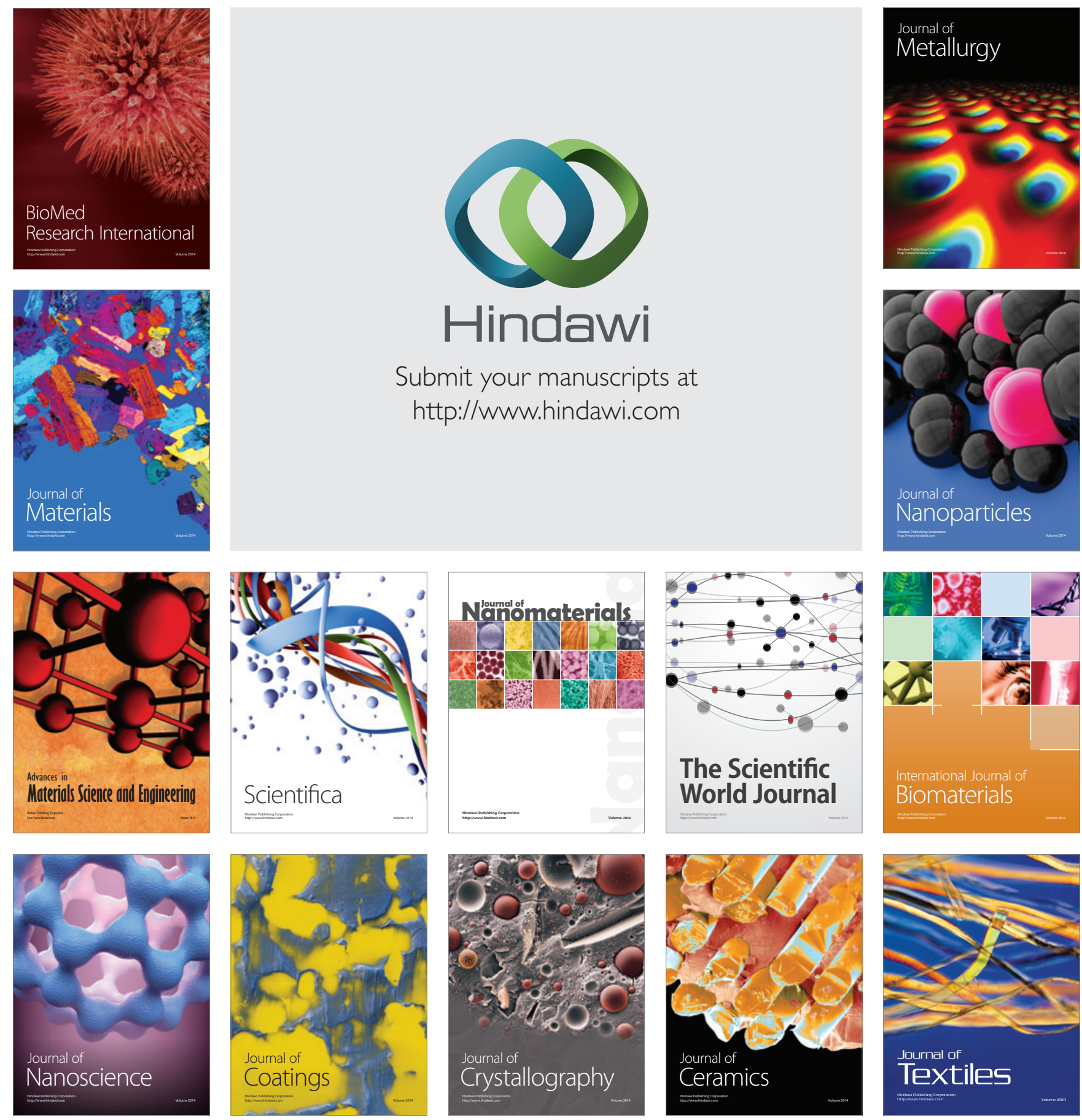